Utah State University

DigitalCommons@USU

$1-1-2011$

\title{
A Field-Widened Spectrometer-Interferometer: Back from the Past to Measure Ionospheric-Thermospheric Energetics
}

Stanley James Wellard

Christopher J. Mertens

Follow this and additional works at: https://digitalcommons.usu.edu/sdl_pubs

\section{Recommended Citation}

Wellard, Stanley James and Mertens, Christopher J., "A Field-Widened Spectrometer-Interferometer: Back from the Past to Measure lonospheric-Thermospheric Energetics" (2011). Space Dynamics Lab Publications. Paper 140.

https://digitalcommons.usu.edu/sdl_pubs/140

This Article is brought to you for free and open access by the Space Dynamics Lab at DigitalCommons@USU. It has been accepted for inclusion in Space Dynamics Lab Publications by an authorized administrator of DigitalCommons@USU. For more information, please contact digitalcommons@usu.edu.

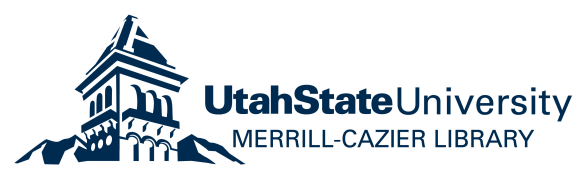




\title{
A field-widened spectrometer-interferometer: Back from the past to measure ionospheric-thermospheric energetics
}

\author{
Stanley James Wellard \\ Space Dynamics Laboratory, 1695 N. Research Park Way, Logan, Utah, USA 84341 \\ Christopher J. Mertens \\ NASA Langley Research Center, Mail Code 401B, Hampton, Virginia 23681
}

\begin{abstract}
Recent broadband observations by the SABER sensor aboard the TIMED satellite hint at intriguing new vibrationrotation excitation and loss processes that occur in the energy dissipation of the ionosphere-thermosphere as it responds to solar storms. To address the questions exposed by the SABER data, SDL's field-widened interferometer has been brought back after three decades to again fly into or above aurorally disturbed atmosphere to gain the data needed to better understand the different processes of ionosphere-thermosphere energetics. The paper discusses the evaluation and design phases (laboratory evaluation, a rocket flight, and a satellite flight) needed to prepare this elegant and unique interferometer to reach its goal of making high resolution $\left(0.5 \mathrm{~cm}^{-1}\right)$ and wide bandwidth (1300$8000 \mathrm{~cm}^{-1}$ ) measurements of the ionosphere-thermosphere world-wide. Design details of interferometer will be presented along with comparisons between a standard Michelson interferometer and the field-widened sensor to illustrate just how the Bounchareine and Connes field-widened form provides the enhanced performance needed for the new missions. The paper also describes how the improved Inferometer design will leverage advances in modern electronics, detectors, bearing design and software to gain significant improvements in the performance of the upgraded field-widened interferometer-spectrometer when compared to the heritage instrument.
\end{abstract}

Keywords: Interferometer, field-widened, spectrometry, Michelson, SDL, ionosphere, thermosphere, energetics

\section{INTRODUCTION}

At a time in photonics research when research interests are focused primarily on such contemporary topics as quantum dots, nanowire antennas, single photon emission and detection processes, organic light emitting materials, along with other exotic topics, it seems innocuous that plans are under way to refly a 40 -year-old sensor that is intended to do cutting edge research into the mechanics of the energetics of the ionospheric-thermosphere. The sensor is a field-widened Michelson interferometer-spectrometer (designated as RBI-2 for Rocket-Borne FieldWidened Interferometer 2) that was developed by Utah State University's Space Dynamics Laboratory between 1973 and 1983. The sensor, flown a final time in April 1983 [1], was the result of numerous iterations and refinements of the field-widened form over those years. As each improved field-widened interferometer/spectrometer emerged, it was deployed to either Alaska's Poker Flats launch facility or to New Mexico's White Sands missile range to make measurements of either the aurorally excited ionosphere-thermosphere or just the quiet night sky.

This paper describes a three-phase program, now underway, to return the 1983 sensor to flight. The effort is collaboration between a team from Langley Research Center (LaRC) in Hampton, Va., and a team from the Space Dynamics Laboratory (SDL) in Logan, Utah. The first phase is to determine, in the laboratory, the current performance parameters of the RBI-2 interferometer and to engineer improvements that can accomplish the design goals needed for the second phase. The second phase, described in a proposal submitted in June 2011, will prepare the sensor for flight on board a sounding rocket to be launched from Poker Flats sometime between December 2012 and February 2013 when the next solar maximum is expected to peak. A third phase, now in the concept stage, is intended to transition the sensor from a sounding rocket to a satellite-based instrument that can make extended global measurements over a longer period of time.

\section{RBI-2 instrument heritage}

USU/SDL has been actively involved with the development, calibration, and operation of air and space-borne hyperspectral and IR FTS systems for many decades. Much of USU/SDL's early work targeted sounding rocket-based missions. As part of this heritage, in the 1970s and1980s, USU/SDL employed "remarkable engineering skills" to develop "the most notable success" in field-widened infrared interferometry [2]. Beginning in the mid-1980s,

Infrared Remote Sensing and Instrumentation XIX, edited by Marija Strojnik, Gonzalo Paez, Proc. of SPIE Vol. 8154, 815403 - @ 2011 SPIE · CCC code: 0277-786X/11/\$18 · doi: 10.1117/12.895344 
however, a shift to other launch platforms began leaving the sounding rockets behind as launch vehicles. Beginning in 1979, two CIRRIS spectrometers (CIRRIS I and CIRRIS-1A) were integrated onto the shuttles STS-4 and STS-39 respectively to fly in-near earth orbits and collect data at the poles and across the continents of the world. Concurrent with the CIRRIS FTSs flying on shuttles, SPIRIT-I and SPIRIT-II, were launched aboard sounding rockets and SPIRIT-III was flown aboard the MSX satellite in 1992. More recent interferometric based sensors have been the Geosynchronous Imaging Fourier Transform Spectrometer (GIFTS) and the Far-Infrared Spectroscopy of the Troposphere (FIRST). The FIRST sensor flew aboard a high-altitude balloon, and the GIFT sensor is still waiting for its flight aboard a satellite. The following sections describe details of the successful field-widening technique used on RBI-2 and other associated flight campaigns to explain why these interferometers perform so well aboard a sounding rocket.

\section{Historical perspective}

In 1983, "personal computing" was just beginning to replace the mainframes that had previously been the work horses for reducing interferometric data. A hundred years earlier in 1880, A. A. Michelson had invented his interferometer and then, years later, in the early 80s; smaller, dedicated computers became available that could effectively do the fast Fourier Transform (FFT). Out of this union came an instrumentation package that was, and is still, the way that scientists can acquire spectra for study over wavelengths that range from the ultraviolet down to the microwave bands. Scenes of interest have ranged from excited aurora, air glow, nebulas, and planets to air pollution discharging from industrial smoke stacks along with many other areas in between. As Fourier Transform Spectroscopy (FTS) has become a routine method for transforming interference patterns, it has expedited the cataloging of spectra from many different radiation sources and has significantly expanded the body of knowledge needed to understand the different chemical processes that abound in our environment. This gathered information has provided a comprehensive basis for understanding the complex energetics of our environment.

\section{SOME THEORY BEHIND STANDARD AND FIELD-WIDENED MICHELSON INTERFEROMETERS}

\section{Standard Michelson interferometer}

To understand the reasons for choosing a field-widened interferometer to do studies of the energetics of the thermosphere-mesosphere, it is useful to first review some of the properties of the standard Michelson interferometer to see its strengths and shortcomings. The following development is after the work of Lowenstein $[3,4]$, Baker [5, 6] and Steed [7].

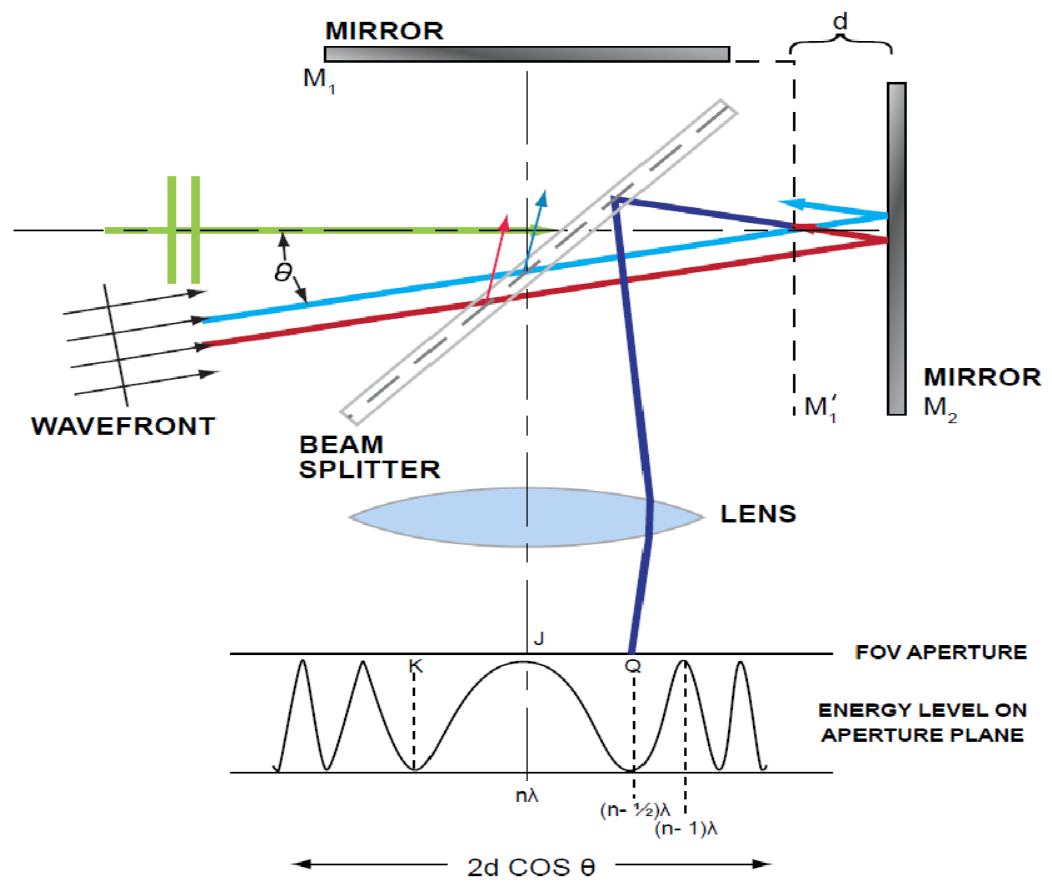

Figure 1. Interaction of off-axis rays in a standard Michelson interferometer. 
Figure 1 shows the geometry of a standard Michelson interferometer. The figure shows plane waves (rays) arriving at the beamsplitter, both normal rays (green) and those at an angle $\theta$ (red and blue). Drive mirror $\mathrm{M}_{1}$ is shown imaged on fixed mirror $\mathrm{M}_{2}$ as $\mathrm{M}_{1}{ }^{\prime}$. Rays, both normal and off-axis, undergo an amplitude division at the beamsplitter with a part of the energy going to the fixed mirror and another equal part going to the moving mirror. After reflection by the mirrors, the energy recombines and interferes either constructive or destructively depending on the optical separation (d) of the two mirrors. The green ray moves through the optics to focus at the center of the focal plane. The red and blue rays combined at the beamsplitter to form one ray (purple) which is focused at point Q at the image plane. Before recombining, the red ray travels a distance $2 \mathrm{~d} \cos (\theta)$ further than does the blue ray. On axis rays, where $\theta$ is zero, have a path difference of just $2 \mathrm{~d} \cos (0)$ or $2 \mathrm{~d}$ which is equal to $\mathrm{n} \lambda$ and produce a maximum because of constructive interference. As the angle of the incident wave increases, the interference becomes less constructive (loss of coherence) until the energy at the focal plane reduces to zero (point Q).

With $d$ set to a value that produces maximum constructive interference, the bottom panel of Figure 1shows the energy distribution as a function of $\theta$ or $2 d \cos (\theta)$. For a given $d$ and $\theta$, there are maxima where $2 d \cos (\theta)$ equals $n \lambda$ and (n-1) $\lambda$ and nulls where $2 d \cos (\theta)$ equals $(n-1 / 2) \lambda$ and (n-3/2) $\lambda$. This variation in energy with $\theta$ results in the observation of circular or Haidinger fringes at the focal plane in the pattern as shown in Figure 1.

For an ideal monochromatic on-axis point source, represented by the normal green ray in Figure 1, interferometric energy reaching the detector can be described by the equation

$$
\mathrm{E}_{\mathrm{det}}=2 \varepsilon \mathrm{B}(\sigma) \mathrm{d} \sigma[1+\cos (2 \pi \sigma \mathrm{d})] .
$$

In this equation, $\varepsilon$ is the efficiency of the beamsplitter, $\mathrm{B}(\sigma) \mathrm{d} \sigma$ is the amplitude of the wave at a spatial frequency $\sigma$ and $\mathrm{d}=\mathrm{x} 1-\mathrm{x} 2$ is the path difference between the two mirrors. The interferogram component of Equation (1) is defined as

$$
\mathrm{d} \mathrm{I}(\mathrm{d})=2 \varepsilon \mathrm{B}(\sigma) \mathrm{d} \sigma[\cos (2 \pi \sigma \mathrm{d})]
$$

When off-axis rays from an extended source are included, Equation (1) becomes

$$
\mathrm{d} \mathrm{E}_{\mathrm{det}}=2 \varepsilon \mathrm{B}(\sigma) \mathrm{d} \sigma[1+\cos (2 \pi \sigma \mathrm{d} \cos (\theta))] \mathrm{d} \Omega,
$$

where $\theta$ is the angle between the off-axis rays and the optical axis and $\mathrm{d} \Omega$ is the differential solid angle. Assuming that $\theta$ will be small enough such that $\cos (\theta)$ can be approximated by $1-\theta^{2} / 2$, Equation (3) becomes

$$
\mathrm{dE}_{\mathrm{det}}=2 \varepsilon \mathrm{B}(\sigma) \mathrm{d} \sigma\left[1+\cos \left(2 \pi \sigma \mathrm{d}\left(1-\theta^{2} / 2\right)\right)\right] \mathrm{d} \Omega .
$$

Setting the phase term, $2 \pi \sigma d\left(\theta^{2} / 2\right)$ in (4) to $\pi$, where $\pi$ is the phase difference between the central ray and the ray that produces the first intensity null, gives

$$
\theta^{2}=1 /(\sigma \mathrm{d})
$$

as the solution for the equation. $\Omega$, the solid angle, subtended from the center to the first minimum, for a given $\sigma$ and $\mathrm{d}$, is then

$$
\Omega=\pi /(\sigma \mathrm{d}) .
$$

For a desired maximum wavenumber, $\sigma_{\max }$, the central fringe continues to decrease in diameter until the retardation reaches a maximum value $L$ where $L$ has been selected to give the desired resolution $(\Delta \sigma=1 / \mathrm{L})$ that will adequately characterize the spatial frequencies of the transformed spectra. Equation 6 then becomes

$$
\Omega_{\max }=\pi /\left(\sigma_{\max } L\right) .
$$

Equation (6) is then the basis for setting the field stop that defines the FOV such that it is approximated the same size as $\Omega_{\max }$.

When extended sources are observed with a standard Michelson interferometer, two other significant effects are introduced into the collected interferograms as the differential energy described by equation (4) is integrated with respect to solid angle. The integration results in the equation

$$
\mathrm{E}_{\mathrm{det}}=2 \varepsilon \mathrm{B}(\sigma) \mathrm{d} \sigma \Omega[1+\operatorname{sinc}(\sigma \mathrm{d} / 2 \pi) \cos (2 \pi \sigma \mathrm{d}(1-\Omega / 4 \pi))] .
$$

The equation shows that the energy of the interferogram is now modulated by a sinc function that describes an attenuation that is a function of the retardation (2d) of the interferometer. It also shows that all wavenumbers are shifted by a factor that depends on the solid angle. This dependency is given by the equation $\sigma_{\text {obs }}=\sigma_{\text {real }}\left(1-\Omega_{\max } / 4 \pi\right)$ ,which says that the observed wavenumber will be lower than the actual wavenumber depending on the value of the field-of-view. 


\section{Field-widening Michelson interferometer}

Equation (6) shows that the maximum-allowable field-of-view for the standard Michelson interferometer depends on the resolution requirements for the system and this has proven to be a major constraint when weak extended sources are to be measured. The need was to find a technique that allowed better throughput and high resolution. Baker [5] provides detailed descriptions of different efforts that were pursued to meet the goal of keeping $\Omega$ large when high resolution was required.

Researchers at SDL focused primarily on the field-widened technique developed by Bouchareine and Connes [8]. This method inserts optical material (two back-silvered, quartz prisms) into the two optical paths of the interferometer for the field compensation. This method was attractive because it had only one moving part, and it was relatively easy to manufacture the required optics.

With appropriate optical material inserted into the optical paths of the interferometer, its retardation changes from $\mathrm{R}$ $=2 \mathrm{~d} \cos (\theta)$ to

$$
R=2 d \cos (\theta)+2 \operatorname{tn} \cos (\alpha)-2 t \cos (\theta)
$$

where

$$
\begin{aligned}
& \mathrm{t}=\text { the thickness of the optical material } \\
& \mathrm{n}=\text { index of refraction for the material } \\
& \theta=\text { angle of off-axis ray } \\
& \alpha=\text { refracted angle of off-axis ray through the optical material. }
\end{aligned}
$$

From Snell's law, $\left.\alpha=\sin ^{-1}(\sin (\theta) / \mathrm{n})\right)$ and the trigonometric series, $\sin (\theta)=\theta-\theta^{3} / 6+\cdots, \sin ^{-1}(\theta)=\theta+\theta^{3} / 6-\cdots, \alpha=$ $\theta / \mathrm{n}=\left(\left(\mathrm{n}^{2}-1\right) \theta^{3}\right) / 6 \mathrm{n}^{3}+\cdots$, and $\cos (\theta)=1-\theta^{2} / 2 !+\theta^{4} / 4 !-\cdots$, the retardation can be rewritten as,

$$
\left.\mathrm{R}=2 \mathrm{~d}\left(1-\theta^{2} / 2 !+\theta^{4} / 4 !\right)+2 \operatorname{tn}\left(1-\theta^{2} / 2 \mathrm{n}^{4}+\left(\theta^{4} / 6 \mathrm{n}\right)(\mathrm{n} 2-1)+\theta^{4} /\left(24 \mathrm{n}^{4}\right)\right)\right)-2 \mathrm{t}\left(1-\theta^{2} / 2 !+\theta^{4} / 4 !\right)
$$

After the expansion and collecting like terms,

$$
\mathrm{R}=2(\mathrm{t}(\mathrm{n}-1)+\mathrm{d})+\mathrm{t}(((\mathrm{n}-1) / \mathrm{n})-\mathrm{d}) \theta^{2}+\left(\mathrm{d} / 12+\left(\left(\left(\mathrm{n}^{2}-1\right) / 3 \mathrm{n}^{3}\right)+\mathrm{t} /\left(12 \mathrm{n}^{3}\right)-\mathrm{t} / 12\right) \theta^{4}\right.
$$

In equation (11), in the second term on the right, the dependence of retardation on $\theta$ can be significantly reduced if $\mathrm{d}$ $=\mathrm{t}((\mathrm{n}-1) / \mathrm{n})$. By changing the thickness of the optical material as the drive distance increases, retardation becomes mostly independent of the $\theta^{2}$ term and the field-widened relationship can be maintained. The new compensated retardation is described by,

$$
\mathrm{R}_{\mathrm{c}}=2(\mathrm{t} / \mathrm{n})\left(\mathrm{n}^{2}-1\right)+\theta^{4}\left(\mathrm{t} / 4 \mathrm{n}^{3}\right)\left(\mathrm{n}^{2}-1\right) .
$$

The retardation is now a function of the instantaneous thickness of the paths through the prisms and the index of refraction of the quartz prisms. The retardation is still proportional to $\theta$ because of the second term of the equation but this effect is small. Physically the field-widened interferometer, a variant of the standard Michelson's interferometer, differs only in that prisms have been substituted for the plane mirrors of the standard interferometer.

\section{New performance parameters after field-widening compensation}

After the interferometer has been compensated per equation (12), there are a number of significant changes in the performance of the compensated interferometer. The field-of-view is now free of the limits imposed by the resolution requirement imposed by the standard interferometer. When the moving prism is positioned properly and equation (12) describes the effective retardation, the limit on the maximum FOV now becomes, in theory, the optical aberrations of the system. Analysis provided by Steed [7] shows that the FOV of a compensated interferometer improves, in theory, by about two orders of magnitude when compared to the FOV of the standard Michelson. The optical aberrations now limiting the FOV are due specifically to chromatic and spherical aberrations along with the astigmatisms of the compensating prisms. In a non-ideal interferometer, the real limit becomes vignetting because it is difficult to process wide fields through a practical optical system without causing vignetting elsewhere in the system.

The next change to accommodate is the longer drive distance needed when the Bouchareine and Connes' technique has been implemented. Again, from Steed [7], the optical path length difference due to the translation of the wedge is calculated to be,

$$
\Delta_{\mathrm{c}}=2 \mathrm{X} \sin (\beta-\gamma)
$$


where $\beta=\sin (n \sin (\alpha))$ and $\gamma=\left(\alpha-\tan ^{-1}(((n-1) / n) \tan (\alpha))\right.$. If $n$ is 1.45 and the wedge angle, $\alpha$, is 8 degrees, then $\beta$ $=11.64$ degrees and $\gamma=5.5$ degrees which makes $\Delta_{c}=0.2 \mathrm{X}$. Since $\Delta_{\mathrm{M}}=2 \mathrm{X}$ for the standard Michelson interferometer and $\Delta_{\mathrm{c}}=0.2 \mathrm{X}$ for a field-widened interferometer, the result shows that a field-widened interferometer has to be driven 5 times farther than does standard Michelson to achieve the same resolution.

\section{Gains made using field-widened interferometers}

Table 1, below, based on a similar table in [6], provides a summary comparison between an uncompensated Michelson interferometer and the field-widened interferometer described above. The comparison is for $\Delta \sigma=2 \mathrm{~cm}^{-1}$ at $\sigma=5000 \mathrm{~cm}^{-1}$ and is used to illustrate, to an order of magnitude, the performance differences to be expected from an interferometer that has been compensated.

The above development has shown that field-widening is a compensation technique that allows, simultaneously, both relatively high spectral resolution and high sensitivity. The improvement in performance comes as energy is collected from a wider range of incident angles when compared to the standard Michelson. A well-compensated field-widened interferometer is well suited to quickly collect high-resolution data in the explosive dynamics of a sounding rocket flight.

Table 1. Comparisons between an uncompensated Michelson interferometer and a field-widened interferometer.

\begin{tabular}{|l|c|c|c|}
\hline & $\begin{array}{c}\text { Uncompensated } \\
\text { Michelson } \\
\text { Interferometer }\end{array}$ & $\begin{array}{c}\text { Field-widened } \\
\text { Interferometer }\end{array}$ & $\begin{array}{c}\text { Change } \\
\text { Ratio }\end{array}$ \\
\hline FOV, Half-angle $\Theta$ (degrees) & 1.25 & & 4.8 \\
\hline Solid Angle, $\Omega=\pi \theta^{2}$ (steradians) & 0.0016 & 0.034 & 23 \\
\hline Relative Drive Distance (cm) & 1 & 5 & 5 \\
\hline $\begin{array}{l}\text { Measurement Time, } \mathrm{T} \sim \Omega^{2} \\
=\pi^{2} \theta^{2} \text { (seconds) }\end{array}$ & 1 & 0.0022 & 454 \\
\hline
\end{tabular}

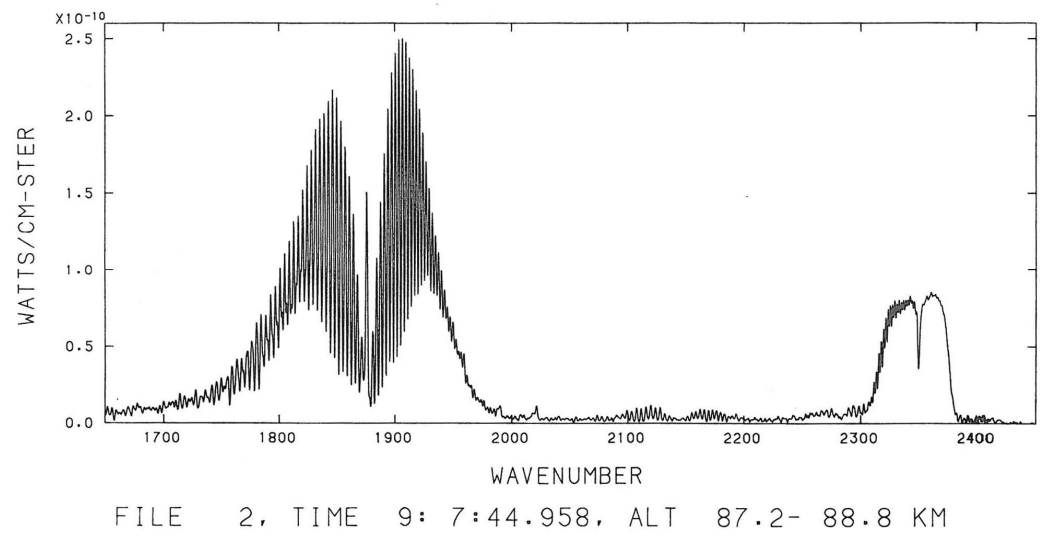

Figure 2. Representative spectral data collected between 87.2 to 88.8 kilometers. The data shows NO bands at $5.4 \mu \mathrm{m}$ $\left(1852 \mathrm{~cm}^{-1}\right)$ and $\mathrm{CO}_{2}$ bands at $4.3 \mu \mathrm{m}\left(2325 \mathrm{~cm}^{-1}\right)$

\section{UPGRADES TO RBI-2 DERIVED FROM SCIENTIFIC REQUIREMENTS}

\section{Early flight RBI-2 campaign}

On 13 April 1983, the Sergeant sounding rocket A30.276 was launched from Poker Flat Research Range in Alaska into an IBC Class II aurora [9, 1]. The RBI-2 field-widened Michelson interferometer/spectrometer that was designed, fabricated, and calibrated at USU/SDL and was the prime instrument aboard the payload [5]. The 
interferometer was designed with high spectral resolution and sensitivity to measure and characterize atmospheric emitters and excitation mechanisms. The flight of A30.276 was a survey mission and provided the first resolved spectra of carbon monoxide (CO) near $4.7 \mu \mathrm{m}$ and of ionized nitric oxide (NO+) near $4.3 \mu \mathrm{m}$. Data from this flight also provide the first high resolution spectra of other atmospheric emitters as a function of altitude such as hydroxyl (OH) near $2.8 \mu \mathrm{m}$, carbon dioxide $\left(\mathrm{CO}_{2}\right)$ near $4.3 \mu \mathrm{m}$ and nitric oxide (NO) near $5.4 \mu \mathrm{m}$ [9, 1]. Typical spectral data, as shown in Figure 2, clearly shows the unique capabilities of a field-widened interferometer and its potential to collect new spectral information to answer compelling questions that have developed from analysis of recent data collected by the Sounding of the Atmosphere using Broadband Emission Radiometry (SABER) instrument on the Thermosphere-Ionosphere-Mesosphere Energetics and Dynamics (TIMED) satellite.

\section{Infrared interferometry of aurorally disturbed ionosphere-thermosphere energetics}

The purpose then for returning the 1983 interferometer/spectrometer to flight is to develop a new prototype fieldwidened Michelson interferometer (FWMI) with enhanced capabilities that can again measure auroral infrared spectra during a storm time enhancement. These measurements, in the auroral region, will augment the SABER data to provide an improved scientific understanding of the fundamental chemical-radiative mechanisms responsible for the response of the ionosphere-thermosphere (IT) to solar-geomagnetic storms.

There is a compelling need to understand the effects of solar-geomagnetic storms since they can disrupt and degrade satellite and ground-based technological systems. Infrared radiation is the primary means by which the atmosphere dissipates energy input into the IT system. Consequently, understanding the physical mechanisms responsible for infrared emission is essential to understanding the energy flow, composition, and dynamics of the upper atmosphere. For example, an improved understanding of the physical mechanisms for storm-time enhancements in infrared emission will lead to better satellite remote sensing methodologies and better large-scale numerical model predictions of thermospheric density perturbations that affect space debris tracking and satellite drag, and ionospheric E-region electron density perturbations that affect radio wave communication and navigation [10, 11, $12,13]$.

\section{Scientific motivation}

The new FWMI, along with other new ancillary sensors, is intended to take data that can help in understanding the recent discoveries by the SABER sensor. The sensor observed large enhancements of thermospheric infrared limb emission in a number of radiometer channels during solar-geomagnetic storm events. For example, the SABER 5.3 $\mu \mathrm{m}$ channel (1840-1980 $\left.\mathrm{cm}^{-1}\right)$ limb radiance measurements were enhanced by factors of 6 to 10 during the April 2002 storm event $[14,15]$. A storm-time enhancement of $\mathrm{NO}(\mathrm{v}) 5.3 \mu \mathrm{m}$ emission is the primary mechanism of dissipating solar storm energy in the thermosphere. Thus, $\mathrm{NO}$ (v) $5.3 \mu \mathrm{m}$ emission is known as a "natural thermostat" by which heat and energy are lost from the thermosphere to space and to the lower atmosphere [14, 15]. However, large uncertainties persist in the role of chemiluminescent emissions, nascent vibrational, rotational, and spin-state distributions, and the role of vibration-rotation and spin-state non-local thermodynamic equilibrium processes [16, $17,18,19,20,21,22,15]$. It will be the role of the rocket-borne FWMI, when it flies at the height of the next sunspot cycle in December 2013 or January 2014, to produce new spectral data similar to that shown in Figure 2. With this new information, the uncertainties associated with the energetics models can be reduced.

\section{Overarching science objectives and supporting questions}

The discoveries from the SABER radiometer have generated three important new science questions which, when answered, will lead to a more complete, physics-based understanding of the excitation and loss mechanisms of the two largest sources of infrared radiative emission in response to solar-geomagnetic storms. The science questions are:

Q1: What are the nascent vibration-rotation distributions of the exothermic chemical reactions forming $\mathrm{NO}^{+}(\mathrm{v})$, especially the dominant $\mathrm{O}_{2}{ }^{+}+\mathrm{NO}$ charge transfer reaction? Since $\mathrm{O}_{2}\left({ }^{1} \Delta\right)$ is formed directly or indirectly from the same charge transfer reaction, knowledge of the vibration-rotation bands of the $\mathrm{O}_{2}\left({ }^{1} \Delta\right)$ electronic transition provides important clues into the details of the chemical-kinetic energy transfer mechanisms associated with the key $\mathrm{O}_{2}^{+}+\mathrm{NO}$ charge transfer reaction.

Q2: What is the nascent vibration-rotation distribution of the $\mathrm{O}_{2}\left({ }^{1} \Delta\right)$ electronic transition?

Q3: What are the nascent vibration-rotation-spin distributions for the exothermic chemical reactions that form $\mathrm{NO}(\mathrm{v})$ ?

\section{Science requirements traceability and proposed instrument development}

New spectral infrared radiance measurements are required to answer the three science questions. The $\mathrm{NO}(\mathrm{v}) 5.3 \mu \mathrm{m}$ bands, including the chemiluminescent emission bands, span the spectral region from $1300-2100 \mathrm{~cm}^{-1}$ [21]. The 
$\mathrm{NO}^{+}(\mathrm{v}) 4.3 \mu \mathrm{m}$ emission bands radiate from $2100-2600 \mathrm{~cm}^{-1}$ [23]. The vibration-rotation bands of the $\mathrm{O}_{2}\left({ }^{1} \Delta\right)$ electronic transition covers the spectral region from 7664-8065 $\mathrm{cm}^{-1}$ [14]. In order to simulate the $\mathrm{NO}(\mathrm{v}), \mathrm{NO}^{+}(\mathrm{v})$, and $\mathrm{O}_{2}\left({ }^{1} \Delta\right)$ bands emissions from a physics-based model, it is useful to derive rotational temperatures for model input from a spectral region relatively insensitive to storm-time enhancements - for example, from the $\mathrm{OH}(\mathrm{v})$ $\Delta \mathrm{v}=1$ Meinel bands near $2.8 \mu \mathrm{m}\left(2900-3600 \mathrm{~cm}^{-1}\right)$ [1]. Furthermore, the $\mathrm{OH}(\mathrm{v}) \Delta \mathrm{v}=2$ Meinel bands near $2.0 \mu \mathrm{m}$ (4400-5220 $\left.\mathrm{cm}^{-1}\right)$ and $1.6 \mu \mathrm{m}\left(5680-6470 \mathrm{~cm}^{-1}\right)$ are used in the non-local thermodynamic equilibrium $\mathrm{CO}_{2}$ model in order to remove the background $\mathrm{CO}_{2}\left(v_{3}\right) 4.3 \mu \mathrm{m}$ emission and isolate the $\mathrm{NO}^{+}(\mathrm{v}) 4.3 \mu \mathrm{m}$ bands [13] The spectral resolution that enables the separation and isolation of the various $\mathrm{NO}^{+}(\mathrm{v}) 4.3 \mathrm{um}$ and $\mathrm{NO}(\mathrm{v}) 5.3 \mu \mathrm{m}$ bands, and the retrieval of rotational temperatures from $\mathrm{OH}(\mathrm{v}) 2.8 \mu \mathrm{m}$ spectral radiance measurements, is on the order of $1.0 \mathrm{~cm}^{-1}$ (unapodized) [1]. Furthermore, in order to achieve the same signal-to-noise quality as the broadband SABER measurements, infrared spectral measurements that can detect a signal as low as $10^{-13} \mathrm{~W} \mathrm{~cm}^{-2} \mathrm{sr}^{-1}\left(\mathrm{~cm}^{-1}\right)^{-1}$ throughout the $1300-8100 \mathrm{~cm}^{-1}$ region are required. In summary, three changes to the 1983 instrument are needed to prepare it to produce the quality data needed to answer the questions found in the SABER data. The three changes are,

(1) an expansion of the spectral bandpass of the sensor from $1250-5000 \mathrm{~cm}^{-1}$ to $1300-8100 \mathrm{~cm}^{-1}$,

(2) an increase in spectral resolution from $\sim 1.2 \mathrm{~cm}^{-1}$ to $\leq 1.0 \mathrm{~cm}^{-1}$, and

(3) a new dynamic range characterized by a $10^{-13} \mathrm{~W} \mathrm{~cm}^{-2} \mathrm{sr}^{-1}\left(\mathrm{~cm}^{-1}\right)^{-1}$ noise equivalent radiance (NER).

These new requirements are essential to answering the three science questions and to the overall science question as listed above.

SABER only provided an integral constraint via its broadband measurements. In order to advance our understanding of IT storm-time energetics beyond the current state of knowledge, high-resolution spectral infrared measurements are needed made with the enhanced FMWI. SABER measurements have expanded the current state of knowledge, but at the same time have approached a knowledge barrier that can only be breached by the new spectral infrared measurement capability we are proposing to develop in the new FWMI.

\section{TECHNICAL APPROACH AND METHODOLOGY}

\section{Phase II payload overview}

To make the needed measurements that can answer the questions derived from the SABER data; we are leveraging the success of the 1983 rocket-borne Michelson interferometer/spectrometer system that was designed by Utah State University / Space Dynamics Laboratory (USU/SDL) in the early 1980s and flown multiple times on sounding rockets. This sensor was designated the Rocket-Borne Field-Widened Interferometer-II (RBI-2). . Utilizing modern electrical designs and current detector technologies, the Phase II sensor, now designated as FWMI (Field-Widened Michelson Interferometer) will be modified in Phase I to have an extended spectral coverage, a higher spectral resolution, an extended dynamical range, and possible reductions in mass, volume, and power. The resultant FWMI will then become the prime sensor that flies in the winter of 2013-2014 to collect the new data that can addresses the key scientific objectives and critical science questions presented in the previous sections.

\section{Field-widened payload description}

Figure 3 shows the RBI-2 interferometer "stacked" in its 1983 flight configuration, with the field-widened interferometer located above its $\mathrm{LN}_{2}$ Dewar, a PCM data system, a metrology laser, and the control electronics and batteries. An atomic oxygen detector, four photometers at $3914 \AA$, and an energy deposition scintillator were also located in the lower section.

There are two interferometers in the stack: the field-widened interferometer, which is the main channel, and an ancillary standard Michelson metrology interferometer to provide the sampling function for the main channel. The air-bearing translation platform is the enabling technology for the field-widened interferometer. Its precise performance is critical to the successful operation of the interferometer. The moving prism, supported by the platform, moved at a high displacement rate $(1.5$ seconds /scan) that optimize the amount of data collected during the flight. At the same time, the platform had to move with great precision and stability, holding an optical tolerance of $\sim 1$ arcsecond over the entire scan. The surfaces of the translation platform and the base were only separated by about 0.0001 inches and the bearing used the boil-off gas from the liquid nitrogen to separate the translation platform from its base. To maintain 0.0001 inch tolerance, the translation platform was fabricated from a custom alloy that had a temperature coefficient that precisely matched the temperature coefficient of the optics [24]. The coefficients of the platform and the prism had to track precisely over a temperature range that varies from $300 \mathrm{~K}$ to $77 \mathrm{~K}$. Holding optical alignment during cool-down and during the vibrations of flight was a stringent requirement. Angular rotation was required to be less than $2 \times 10^{-5}$ radians and any deviation from a straight path was required to be less than $10^{-5}$ $\mathrm{cm}$. 
The beamsplitter was a cube type with halogenated chlorofluoralocarbon oil used as the bonding agent between the two surfaces. This oil was selected because it remained pliable at nitrogen temperatures, it had about the same temperature coefficient as did the calcium fluoride material used to fabricate the beamsplitter, and it was free of absorptions from 1250 to $10,000 \mathrm{~cm}^{-1}$.

The heritage sensor was equipped with two cryogenic Dewars. The first was a liquid neon Dewar that housed the main channel detector. This Dewar had a nominal temperature of $27 \mathrm{~K}$ and a capacity of 1.5 liters. Its hold time was about 48 hours. A second larger $\mathrm{LN}_{2}$ Dewar provided cooling at $77 \mathrm{~K}$ for the interferometers and boil-off gas for the air bearing. This Dewar held 15 liters and had a hold time of about 30 hours.

The main channel detector was fabricated from indium-doped silicon that was located at the focus of a parabolic reflector. Detectors of the type used on the 1983 instrument are no longer available and so, in Phase I, one primary task is to find a suitable prototype replacement detector that is both compatible with the throughput properties of the RBI-2 and capable of meeting the new NER requirement of $10^{-13} \mathrm{~W} \mathrm{~cm}^{-2} \mathrm{sr}^{-1}\left(\mathrm{~cm}^{-1}\right)^{-1}$. Procurement is currently underway to buy a prototype detector and amplifier from IR Associates in Stuart, Fla., and then have TeledyneJudson Technologies in Montgomeryville, Pa., install the IR Associates devices in a custom $\mathrm{LN}_{2}$ Dewar.

The separate helium-neon laser metrology interferometer $\left(0.6328 \mu \mathrm{m}\right.$ or $\left.15803 \mathrm{~cm}^{-1}\right)$ is designed using two corner cube reflectors and a cubic beamsplitter. The detector for the metrology interferometer is a common silicon detector capable of operating at the ambient $77 \mathrm{~K}$ temperature of the $\mathrm{LN}_{2}$ Dewar. This detector is still operational and will be used in the Phase I implementation. The moving corner cube reflector is attached to the main channel interferometer's translation platform and moves in sync with the moving prism. The helium-neon laser is mounted in the electronics bay beneath the $\mathrm{LN}_{2}$ Dewar. The beam is transmitted up through a tube that runs between the warm electronics bay and the cold environment surrounding the main channel interferometer. Hermetic windows on each end of the tube are used to maintain vacuum in the Dewar.

The original electronics for the RBI-2 design are shown in Figure 3. These electronics were not recover and will need to be replaced in Phases' I and II. In phase I the electronics needed to control the movement of the prism translation platform and to do the signal processing for the two interferometer channels will be a combination of hard-wired electronics and a software defined interferometer controller (SDIC). Housekeeping data will be directly input into the LABVIEW system. In Phase II, all hardwired and software defined designs developed in phase I, will be transitioned to space-rated circuits as preparation for the rocket flight.

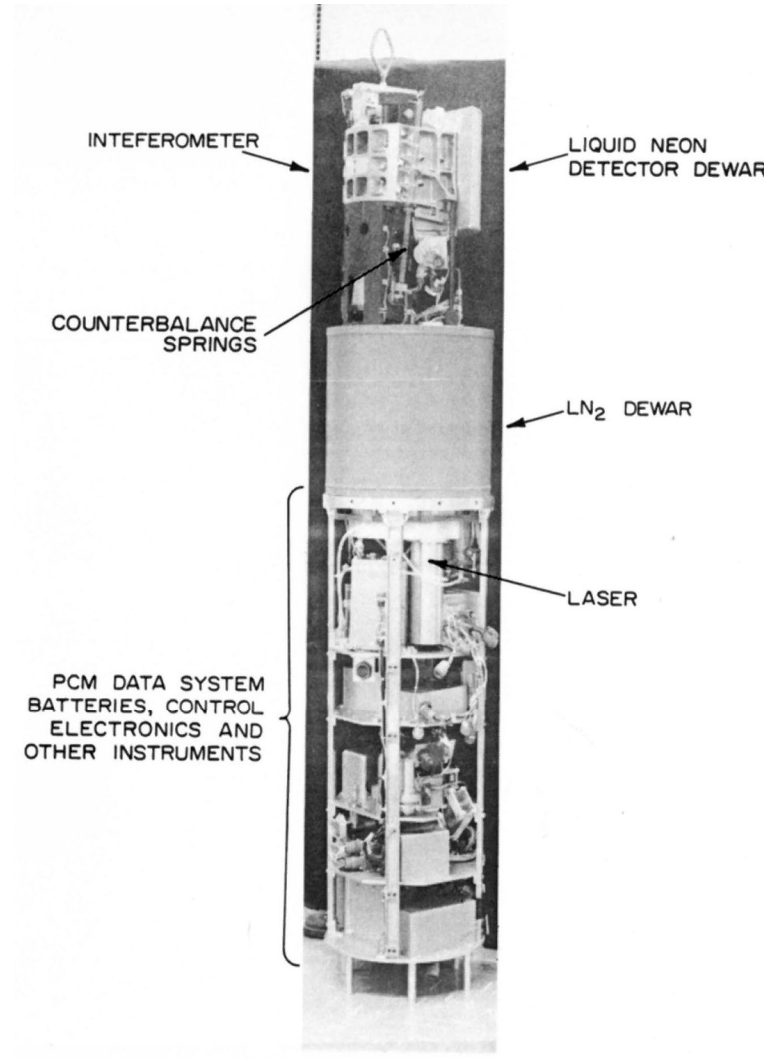

Figure 3. The field-widened interferometer and liquid neon Dewar "stacked" above the PCM data system, system batteries, control electronics, and ancillary instruments.

\section{PHASE III CONCEPTS}

The concept for phase III comes from the expectation that the phase II sensor can further upgraded to fly a satellite mission. The transition would require major changes to the basic instrument that would include the replacement of the air bearing with perhaps an electromagnetic bearing that could support the moving prism for the long duration of a satellite flight along with new cooling systems for the interferometer and for the detector module. It is very probable that cryo-cooler technology, passive cooling, or a combination of the two could successfully replace the existing cold gas based system. The ability for a field-widened interferometer to take rapidly take data should also negate much of the need for the usual sophisticated pointing system needed by the standard interferometer to remain on target during its scan. A satellite-based field-widened instrument is entirely feasible and would allow scientists to measure IT energetics world-wide and, specifically, to focus on measurements made at the two poles, to understand how energetic conditions change over the course of upcoming solar cycles.

\section{Conclusions}

The successful flight of RBI-2 established a solid foundation for the development of the prototype FWMI. New designs and modern technologies will be added to 
the legacy design to upgrade it to accomplish the stated science objectives that are the path to a better understanding of the energetics of the ionospheric-thermosphere. The effort in Phase I is focuses on the development of a new main channel detector system, a new signal conditioning system, and a controller that can increase the displacement of the slide to improve spectral resolution. The heritage field-widened interferometer is the enabling basis for the transition to the FWMI, and this improved instrument will ready to fly in the next solar maximum. It is fully expected that the field-widened interferometer, this elegant variation of the Michelson interferometer, will once again successfully measure the energetics of an auroral disturbed ionosphere-thermosphere and expand the understanding of the complex physics found in the energetics of the ionosphere-thermosphere.

\section{REFERENCES}

[1] Espy. P. J., Harris, C. R., Steed, A. J., Ulwick, J. C. and Haycock, R. H., "Rocketborne interferometer measurement of infrared auroral spectra," Planet. Space Sci., 36, 542 (1988).

[2] Shepherd, G. G., [Spectral Imaging of the Atmosphere], International Geophysics Series 82, Academic Press, San Diego (2002).

[3] Loewenstein, E.V., "The history and current status of Fourier transform spectroscopy," Appl. Optics 5(5) 845-833 (1966)

[4] Loewenstein, E.V., "Fourier spectroscopy: an introduction," Aspen Int. Conf. on Fourier Spectroscopy, 1970. G.A. Vanasse, A.T. Stair, Jr., and D. J. Baker, Eds., U.S. Air Force, L.G. Hanscom Field, Bedford MA, Vol. AFCRL-71-0019 (1971).

[5] Baker, D., Steed, A. and Stair, Jr., A. T., "Development of infrared interferometry for upper atmospheric emission studies," Appl. Opt. 22(10), 1734-1746 (1981).

[6] Baker, D., "Field-widened interferometers for Fourier spectroscopy," chap.2 [Spectrometric Techniques], v. 1, George A. Vanasse, ed., Academic Press, New York (1977).

[7] Steed, A.J., "Field-widened interferometer," Dissertation, Utah State University (1979).

[8] Bouchareine, P. and Connes, P., "Interferometer with compensated field for Fourier transform spectroscopy," J. Phys. Rad. 24, 134-138 (1963).

[9] Picard, R. H., Winick, J. R., Sharma, R. D., Zachor, A. S., Espy, P. J., and Harris, C. R., "Interpretation of infrared measurements of the high-latitude thermosphere from a rocket-borne interferometer," Adv. Space Res., 7(10), 23-30 (1987).

[10] Storz, M. F., Bowman, B. R., and Branson, Maj. J. I. "High accuracy satellite drag model (HASDM)," Paper AIAA-2002-4886 presented at AIAA/AAS Astrodynamics Specialist Conf., Am. Inst. Aeronaut. Astronaut., Monterey, Calif. (2002).

[11] Bruinsma, S. L. and Forbes, J. M. "Medium- to large-scale density variability as observed by CHAMP," Space Weather, 6, S08002, doi:10.1029/2008SW000411 (2008).

[12]Deng, Y. and Ridley, A. J., "Possible reasons for underestimating Joule heating in global models: E field variability, spatial resolution, and vertical velocity," J. Geophys. Res. 112, A09308, doi:10.1029/2006JA012006 (2007).

[13] Mertens, C. J., Mast, J. C., Winick, J. R., Russell, J. M. III, Mlynczak, M. G. and Evans, D. S., "Ionospheric E-region response to solar-geomagnetic storms observed by TIMED/SABER and application to IRI stormmodel development," Adv. Space Res. 39, 715-728 (2007).

[14] Mlynczak, M. G., Martin-Torres, F. J., Russell, J., Beaumont, K., Jacobson, S, Kozyra, J., Lopez-Puertas, M., Funke, B., Mertens, C., Gordley, L., Picard, R., Winick, J., Wintersteiner, P. and Paxton, L. "The natural thermostat of nitric oxide emission $5.3 \mu \mathrm{m}$ in the thermosphere observed during the solar storms of April 2002," Geophys. Res. Lett., 30(21), 2100, doi: 10.1029/2003GL017693 (2003).

[15] Mlynczak, M. G., F. J. Martin-Torres, G. Crowley, D. P. Kratz, B. Funke, M. Lopez-Puertas, J. M. Russell III, J. Kozyra, C. Mertens, R. Sharma, L. Gordley, D. Picard, J. Winick, and L. Paxton), "Energy transport in the thermosphere during the solar storms of April 2002," J. Geophys. Res., 110, A12S25, doi:10.1029/2005JA011141(2005).

[16] Sharma, R. D. and Duff, J. W., "Determination of the translational temperature of the high altitude terrestrial thermosphere from the rotational distribution of the 5.3 um emission from $\mathrm{NO}(\mathrm{v}=1)$," Geophys. Res. Lett. 24, 2407(1997).

[17] Sharma, R. D., Dothe, H. and Duff, J. W. "Model of the 5.3 um radiance from NO during the sunlit thermosphere," J. Geophys. Res. 103(14)753-758 (1998).

[18] Funke, B. and Lopez-Puertas, M., "Nonlocal thermodynamic equilibrium vibrational, rotational, and spin state distribution of $\mathrm{NO}(v=0,1,2)$ under quiescent atmospheric conditions," J. Geophys. Res., 105(D4), 4409-4426 (2000). 
[19] Dothe, H., Duff, J. W., Sharma, R. D. and Wheeler, N. B., "A model of odd nitrogen in the aurorally dosed nighttime terrestrial thermosphere,” J. Geophys. Res., 107(A6), 1071, doi:10.1029/2001JA000143 (2002).

[20] Gardner, J. L., Lopez-Puertas, M., Funke, B., Miller, S. M , Lipson, S. J. and Sharma, R. D., "Rotational and spin-orbit distributions of NO observed by MIPAS/ENVISAT during the solar storm of October/November 2003,” J. Geophys. Res. 110, A09S34, doi:10.1029/2004JA010937 (2005).

[21] Duff, J. W., Dothe, H. and Sharma, R. D. "A first-principles model of spectrally resolved 5.3 um nitric oxide emission from aurorally dosed nighttime high-altitude terrestrial thermosphere," Geophys. Res. Lett., 32, L17108, doi:10.1029/2005GL023124 (2005).

[22] Campbell, L. and Brunger, M. J, "Electron impact contribution to infrared NO emissions in auroral conditions," Geophys. Res. Lett., 34, L22102, doi:10.1029/2007GL031743 (2007).

[23] Mertens, C. J., Winick, J. R., Russell, J. M. III, Mlynczak, M. G., Evans, D. S., Bilitza, D. and Xu, X. "Empirical storm-time correction to the international reference ionosphere model E-region electron and ion density parameterizations using observations from TIMED/SABER," Proc. SPIE, Remote Sensing of Clouds and the Atmosphere XII, Florence, Italy, Sept. 17-19, Vol. 6745, 65451L, doi:10.1117/12.737318 (2007).

[24] Haycock, R.H., "A cryogenically cooled field-widened interferometer for rocket-borne near-infrared atmospheric studies," Space Dynamics Laboratory report, SDL document number SDL/83-029 (1983). 